\title{
Battle over CCL2 for control of the metastatic niche: neutrophils versus monocytes
}

\author{
Jonathan B Mitchem and David G DeNardo*2,3
}

\begin{abstract}
Tumor-derived factors, such as proinflammatory cytokines, can increase the hospitality of metastatic sites by recruiting and activating leukocytes to perform supporting roles during metastatic dissemination. These same cytokines, however, are natural danger signals for the immune system and as such can induce anti-tumor immune responses by both adaptive and innate immune cells. The outcome of tumor-derived inflammatory cytokines is probably closely related to the exact repertoire of factors produced by each tumor. Several recent studies have investigated these seemingly contradictory roles of tumor-derived CCL2 with significant clinical implications.
\end{abstract}

In recent years, significant progress has been made in our understanding of the events that take place during the metastatic process. We have begun to appreciate that several rate-limiting steps in the metastatic cascade such as invasion, extravasation, and survival at the secondary site - are regulated by an intimate interplay of disseminated cells and the local environment present both at the primary site and at the site of metastatic development. Together these cell extrinsic factors collaborate with pre-existing oncogenic and virulence programs in malignant cells to promote successful metastasis. For example, tumor-derived TNF $\alpha$, transforming growth factor beta (TGF $\beta$ ), and Toll-like receptor 4 ligands lead to recruitment of inflammatory cells to the pre-metastatic lung, which in turn enhance metastatic cell extravasation and survival through expression of factors such as S100A3/4, matrix metallopeptidase 9 and vascular endothelial growth factor [1-3]. However, inflammatory cytokines such as these can also activate immune surveillance and induce tumoricidal activity in

*Correspondence: ddenardo@dom.wustl.edu

2Department of Medicine, Washington University School of Medicine,

660 South Euclid Avenue, Box 8069, St Louis, MO 63110, USA

Full list of author information is available at the end of the article the innate immune compartment. This suggests that the exact context or milieu of tumor-derived cytokines may in the end control the hospitality of the metastatic site during and/or prior to metastatic colonization.

A recent example of immune preparation of the premetastatic site by Pollard and colleagues utilized the MMTV-PyMT mouse model of luminal breast cancer and demonstrated that $\mathrm{CD} 11 \mathrm{~b}^{+} \mathrm{CSF} 1 \mathrm{R}^{+} \mathrm{Gr} 1^{+} \mathrm{Ly} 6 \mathrm{C}^{+} \mathrm{CCR} 2^{+}$ inflammatory monocytes are recruited to the premetastatic lung during the process of metastatic colonization by CCL2 [4]. In turn these inflammatory monocytes increase tumor cell extravasation from the vasculature into the lung parenchyma by increasing vascular endothelial growth factor-induced vascular permeability and subsequent malignant cell transendothelial migration. Additionally myeloid cells present in the metastatic site may enhance the survival of extravasated cells by engagement of vascular cell adhesion molecule- 1 [5]. These experiments indicate that CCL2 synthesized by metastatic tumor cells and/or the target-site stroma is critical for the recruitment of a subpopulation of CCR2expressing monocytes that enhance the subsequent extravasation of the tumor cells.

However, recruitment of inflammatory monocytes is not the only consequence of tumor-derived CCL2. Work by Benezra and colleagues has recently shown that tumor $\mathrm{CCR}_{2}{ }^{+}$neutrophils are also recruited to the premetastatic lung [6]. Instead of facilitating metastatic tumors, however, these neutrophils diminish the survival of disseminated cells through CCL2-dependent activation of $\mathrm{H}_{2} \mathrm{O}_{2}$-mediated killing. These results would suggest that inflammatory cytokines such as CCL2 both promote and hinder metastatic tumor onset. The challenge for developing such potential targets clinically is therefore understanding how these processes are concomitantly regulated and when pro-tumor and anti-tumor activity is the dominant player. Perhaps the devil is in the detail. Several tumor-derived cytokines can activate neutrophil cytotoxic activities, including CCL2, CCL5, CCL3, and CXCL1; however, other tumor-derived factors such as granulocyte colony-stimulating factor block potential antitumor neutrophil activity and instead trigger granulocytes to favor tumor growth and metastasis promotion. 


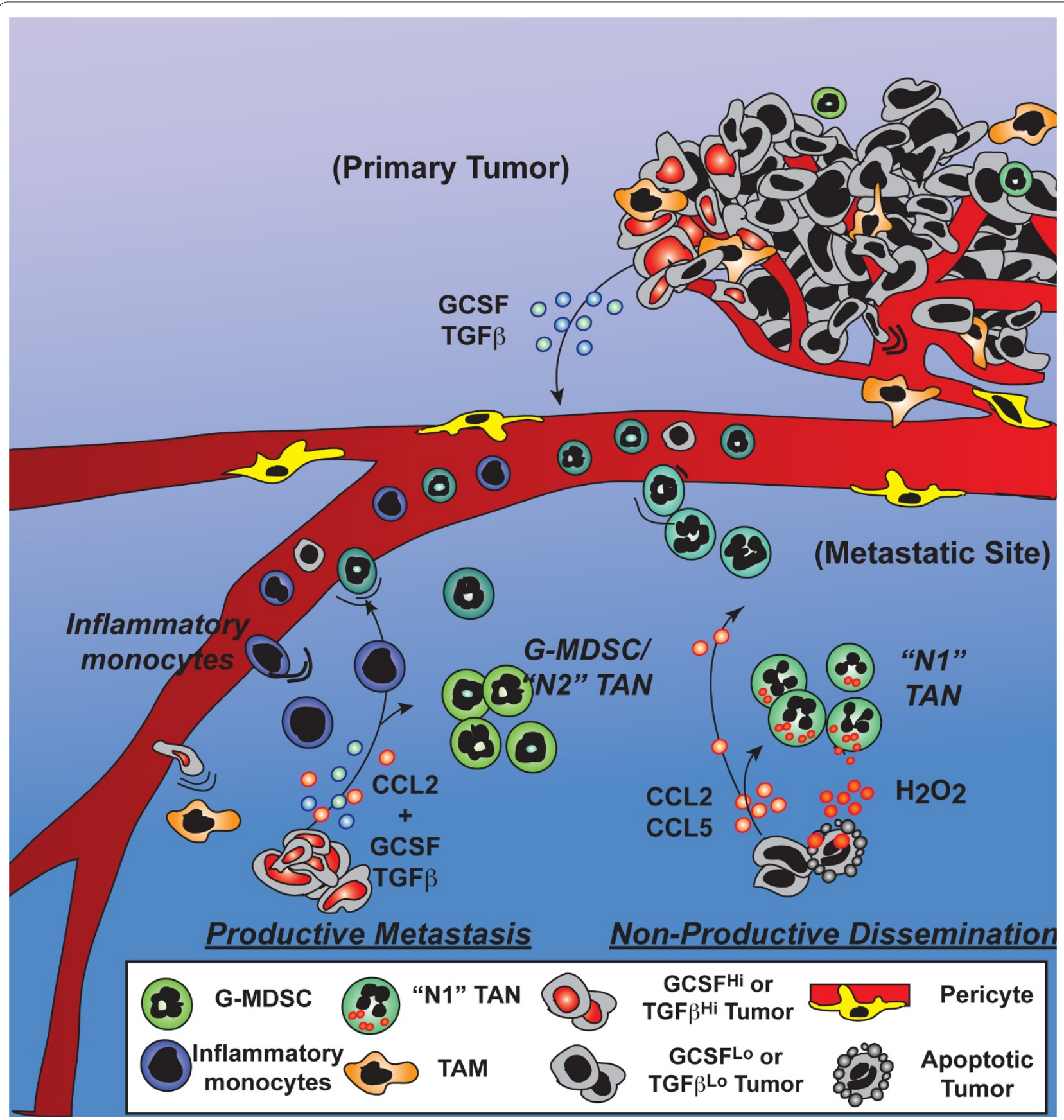

Figure 1. Diverse outcomes of tumor-derived CCL2. CCL2 expression by mammary tumors can recruit and activate circulating tumor-activated neutrophils ('N1'TANs) to kill disseminated tumor cells. However, when these tumors are also producing factors such as transforming growth factor beta (TGF $\beta$ ) and granulocytic colony-stimulating factor (GCSF), immature granulocytes such as granulocyte myeloid-derived suppressor cells (G-MDSC), 'N2'TANs and inflammatory monocytes are preferentially recruited in response to CCL2, and these cells in turn favor disseminated cell survival and proliferation. TAM, tumor-associated macrophage.

Similarly, Fridlender and colleagues demonstrated that when TGF $\beta$ signaling is actively engaged, CD11b+Ly6G ${ }^{\mathrm{Hi}}$ granulocyte precursors promote mammary tumor progression; however, in the presence of inhibition of type I TGF $\beta$ receptor signaling, CD $11 \mathrm{~b}^{+} \mathrm{Ly} 6 \mathrm{G}^{\mathrm{Hi}}$ differentiated neutrophils/granulocytes exhibit tumoricidal activity
[7,8]. Additionally, in another model of breast cancer, Kowanetz and colleagues showed that $\mathrm{Ly}^{6} \mathrm{C}^{+} \mathrm{Ly}_{6 \mathrm{G}}{ }^{+}$ granulocytes were pro-metastatic when mobilized using granulocyte colony-stimulating factor or Bv8 [9].

Taken together these data suggest that metastatic competency may also be tied to the ability of disseminated 
tumors to alter granulocyte differentiation/activation to favor tumor cell survival in response to inflammatory cytokines such as CCL2. The exact milieu of cytokines produced by the primary tumor, as well as disseminated tumor cells, and the composition of the microenvironment at the site of disseminated cell arrest can thus vastly influence the type of immune response elicited (Figure 1). So while expression of CCL2, probably in concert with CCL5, might mediate tumor rejection, CCL2 in concert with TGF $\beta$ may mediate myeloid-derived suppressor cell and/or inflammatory monocyte promotion of disseminated malignant cell survival.

\section{Clinical implications}

While the exact effects of CCL2 on individual immune cell pro-tumor and anti-tumor properties are still being understood, clinical therapeutics targeting CCL2 or CCR2 are currently being employed. Correlative studies in breast cancer and prostate cancer have shown that increased tumor or serum CCL2 levels are associated with worse prognosis [10]. There are therefore currently several registered trials using either anti-CCL2 or antiCCR2 pharmaceuticals for the treatment of solid tumors, in particular prostate cancer. Two of these trials are in patients with advanced disease in combination with standard of care chemotherapy (NCT01204996) and in patients with any primary bony metastases (NCT01015560). Clearly, given the currently available data, CCL2/CCR2 signaling is a worthwhile target in the treatment of human cancers, including breast cancer; however, more work on the correct timing and proper utilization of this signaling may be warranted in order to maximize the efficacy of adding this treatment regimen.

\section{Abbreviations}

CCL2, chemokine ( $\mathrm{C}-\mathrm{C}$ motif) ligand 2; CCR2, chemokine ( $\mathrm{C}-\mathrm{C}$ motif) receptor 2; CSF1R, colony-stimulating factor-1 receptor; MMTV, mouse mammary tumor virus; PyMT, polyomavirus middle T; TGF $\beta$, transforming growth factor beta; TNF, tumor necrosis factor.

Competing interests

The authors declare that they have no competing interests.

\section{Author details}

'Department of Surgery, Washington University School of Medicine, 660 South Euclid Avenue, Box 8109, St Louis, MO 63110, USA. ²Department of Medicine, Washington University School of Medicine, 660 South Euclid Avenue, Box 8069, St Louis, MO 63110, USA. ${ }^{3}$ Department of Pathology/Immunology, Washington University School of Medicine, 660 South Euclid Avenue, Box 8069, St Louis, MO 63110, USA.

Published: 19 July 2012

\section{References}

1. Kaplan RN, Riba RD, Zacharoulis S, Bramley AH, Vincent L, Costa C, MacDonald DD, Jin DK, Shido K, Kerns SA, Zhu Z, Hicklin D, Wu Y, Port JL, Altorki N, Port ER, Ruggero D, Shmelkov SV, Jensen KK, Rafii S, Lyden D: VEGFR1-positive haematopoietic bone marrow progenitors initiate the pre-metastatic niche. Nature 2005, 438:820-827

2. Wels J, Kaplan RN, Rafii S, Lyden D: Migratory neighbors and distant invaders: tumor-associated niche cells. Genes Dev 2008, 22:559-574.

3. Psaila B, Lyden D: The metastatic niche: adapting the foreign soil. Nat Rev Cancer 2009, 9:285-293.

4. Qian BZ, Li J, Zhang H, Kitamura T, Zhang J, Campion LR, Kaiser EA, Snyder LA, Pollard JW: CCL2 recruits inflammatory monocytes to facilitate breasttumour metastasis. Nature 2011, 475:222-225.

5. Chen Q, Zhang XH, Massague J: Macrophage binding to receptor VCAM-1 transmits survival signals in breast cancer cells that invade the lungs. Cancer Cell 2011, 20:538-549.

6. Granot Z, Henke E, Comen EA, King TA, Norton L, Benezra R: Tumor entrained neutrophils inhibit seeding in the premetastatic lung. Cancer Cell 2011, 20:300-314.

7. Fridlender ZG, Buchlis G, Kapoor V, Cheng G, Sun J, Singhal S, Crisanti MC, Wang LC, Heitjan D, Snyder LA, Albelda SM: CCL2 blockade augments cancer immunotherapy. Cancer Res 2010, 70:109-118.

8. Fridlender ZG, Sun J, Kim S, Kapoor V, Cheng G, Ling L, Worthen GS, Albelda SM: Polarization of tumor-associated neutrophil phenotype by TGF-beta: 'N1'versus 'N2'TAN. Cancer Cell 2009, 16:183-194.

9. Kowanetz M, Wu X, Lee J, Tan M, Hagenbeek T, Qu X, Yu L, Ross J, Korsisaari N, Cao T, Bou-Reslan H, Kallop D, Weimer R, Ludlam MJ, Kaminker JS, Modrusan Z, van Bruggen N, Peale FV, Carano R, Meng YG, Ferrara N: Granulocytecolony stimulating factor promotes lung metastasis through mobilization of Ly6G+ ${ }^{+}$LyC $^{+}$granulocytes. Proc Natl Acad Sci U S A 2010, 107:21248-21255.

10. Soria $G$, Ben-Baruch $A$ : The inflammatory chemokines CCL2 and CCL5 in breast cancer. Cancer Lett 2008, 267:271-285.

doi:10.1186/bcr3149

Cite this article as: Mitchem JB, DeNardo DG: Battle over CCL2 for control of the metastatic niche: neutrophils versus monocytes. Breast Cancer Research 2012, 14:315. 